PAPER

\section{Experimental data on low energy electron impact ionisation of $\mathrm{W}$}

To cite this article: T Schlummer et al 2017 Phys. Scr. 2017014075

View the article online for updates and enhancements.
Related content

- ERO modeling of Cr sputtering in the
$\frac{\text { linear plasma device PSI-2 }}{\text { A Eksaeva, D Borodin, A Kreter et al. }}$
- Spectroscopic determination of inverse
$\frac{\text { photon efficiencies of W atoms in the }}{\text { Scrape-off laver of TEXTOR }}$
S Brezinsek, M Laengner, J W Coenen et
al.
- Modelling of plasma-wall interaction and
impurity transport in fusion devices and
prompt deposition of tungsten as
application
A Kirschner, D Tskhakaya, S Brezinsek et
al.




\title{
Experimental data on low energy electron impact ionisation of $\mathbf{W}$
}

\author{
T Schlummer, S Ertmer, S Brezinsek $\bullet$, A Eksaeva, A Pospieszczyk, \\ D Borodin, A Kirschner ${ }^{\circledR}$, A Kreter, Ch Linsmeier ${ }^{-}$, G Sergienko and \\ B Unterberg
}

Forschungszentrum Jülich GmbH, Institut für Energie- und Klimaforschung-Plasmaphysik, Partner of the Trilateral Euregio Cluster (TEC), D-52425 Jülich, Germany

Received 25 July 2017, revised 8 October 2017

Accepted for publication 10 October 2017

Published 28 November 2017

\begin{abstract}
We present experimental data on the rate coefficient for electron impact ionisation of $\mathrm{W}$ atoms at electron temperatures between 5 and $10 \mathrm{eV}$. Our results undercut different theoretical data sets based on the Born approximation and the distorted wave approximation, respectively, by more than a factor of 3. Better agreement in absolute values is found with a more recent work by Goswami et al. The present data match well with earlier measurements by Steinbrink obtained with a different method.
\end{abstract}

Keywords: tunsgten, W, ionisation, cross section, spectroscopy

(Some figures may appear in colour only in the online journal)

\section{Introduction}

Nuclear fusion is one potential option to provide a $\mathrm{CO}_{2}$-free source of electricity in the future. A promising way to realise fusion in hot hydrogen plasmas is with so-called magnetic confinement. Magnetic fusion devices called tokamaks or stellarators use strong magnetic fields to keep the charged plasma particles from recombining at the vessel walls. However, the magnetic confinement is never perfect so that the first wall elements are exposed to high heat loads. In order to permit both flexible operation and high availability of future reactors the selection of the plasma facing components is cruxial. Tungsten (W) is to date the best candidate to handle the high heat loads impinging on first wall elements of fusion plasma devices. It is successfully used in current tokamaks like JET or AUG and is foreseen for future machines where the conditions will be even more demanding [1]. However, due to its high nuclear charge number $\mathrm{W}$ can significantly degrade the plasma performance and energy confinement when abundant as intrinsic impurity. Therefore, a thorough survey and control of the $\mathrm{W}$ concentration in the plasma core is of crucial importance [2]. A key step for understanding the $\mathrm{W}$ behaviour in the plasma is to quantify the $\mathrm{W}$ source at the plasma edge, which can readily be accessed spectroscopically on the basis of SX/B values [3]. Nevertheless, detailed modelling of the relevant processes in the plasma necessitates the knowledge of the rate coefficients for electron impact ionisation of $\mathrm{W}$ and its ionisation stages. There are several publications giving theoretical data on the cross sections or rate coefficients for near threshold electron impact ionisation of $\mathrm{W}^{0}$, of which the most widely used are those by Vainstein et al [4] and ADAS [5]. Vainstein provides a parametrised expression for the rate coefficients obtained by fitting his data:

$$
\begin{gathered}
\left\langle\sigma_{\text {ion }} \cdot v\right\rangle=10^{-8} \cdot A \\
\cdot \frac{\sqrt{\frac{R y}{T_{e}}} \cdot\left(\frac{R y}{T_{e}}+1+D\right)}{\left(\frac{R y}{T_{e}}+X\right) \cdot\left(\frac{R y}{T_{e}}+1\right) \cdot \sqrt{\left(\frac{E_{i z}}{T_{e}}+1\right)}} \cdot \mathrm{e}^{\frac{-E_{i z}}{T_{e}}},
\end{gathered}
$$

where $A=60.4, X=0.048, \quad D=-0.3, R y=13.6 \mathrm{eV}$ and $E_{i z}=7.86 \mathrm{eV}$. There is also one experimental data set by Steinbrink [6] for electron temperatures between 2 and $18 \mathrm{eV}$, which undercuts the theoretical ones significantly. The Steinbrink data are described well by expression 1 with $A=43.6959, X=4.27368, D=0.30302$.

In this work we present new experimental results on the ionisation rate coefficient for electron impact ionisation of W0 at near threshold temperatures obtained spectroscopically at the linear plasma device PSI-2. Further details regarding this work can be found in [7]. 


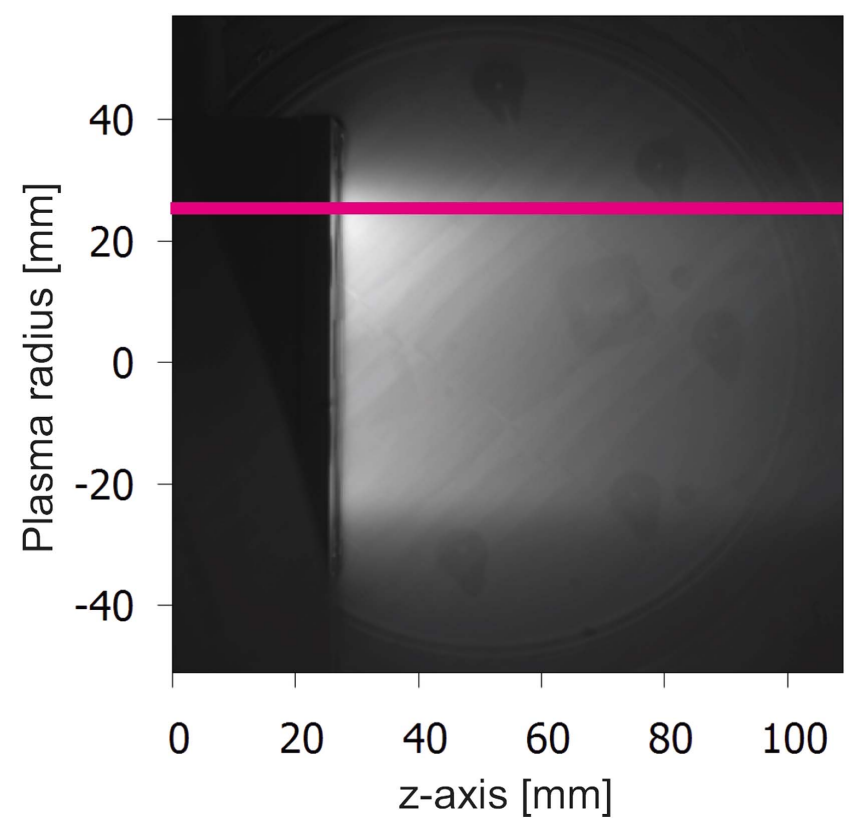

Figure 1. Filter camera image of W I line emission $(400.9 \mathrm{~nm})$ in front of a biased target. The magenta line indicates the plasma volume covered by the imaging spectrometer. The sightline is oriented into the plane of projection.

\section{Experimental setup}

PSI-2 [8] is a linear plasma device optimised for plasmamaterial interaction studies under divertor-like conditions. Achievable electron temperatures and densities range between $1-30 \mathrm{eV}$ and $10^{16}-10^{18} \mathrm{~m}^{-3}$, respectively, depending on the working gas. With a magnetic field of $100 \mathrm{mT}$ the plasma column reaches a diameter of approx. $6 \mathrm{~cm}$ while electron temperature and density show hollow profiles.

An axial target manipulator allows for moving different types of target plates freely along the $z$-axis. For the present experiment a W target plate $\left(8 \times 10 \mathrm{~cm}^{2}\right)$ covering the entire plasma diameter is exposed to a number of pure neon plasmas with differing discharge powers while kept at a constant bias voltage of $-200 \mathrm{~V}$-enough to allow tungsten sputtering by neon ions for which the threshold energy is approx. $40 \mathrm{eV}$ [9].

We use a Czerny-Turner spectrograph to observe the spectral emission of the sputtered $\mathrm{W}$ atoms with spatial resolution in the $z$-direction and at different wavelengths. The sightline covers approx. $10 \mathrm{~cm}$ in front of the target plate intersecting the plasma volume in the upper peak (figure 1) and has a width of $<1 \mathrm{~mm}$ in radial direction.

The sputtered atoms coat the surface of the optical window used for the spectroscopic survey with a thin, slowly growing $\mathrm{W}$ layer. This leads to a time dependant degradation of the window's transmissivity. Also the layer thickness shows a gradient in $z$-direction due to the angular distribution of the sputtered particles. In order to correct for this effect the window's transmissivity is thoroughly monitored with spatial resolution and for all relevant wavelengths using a reference light source in between subsequent plasma scenarios.

The electron density and temperature as well as the plasma potential are determined by means of a single Langmuir probe

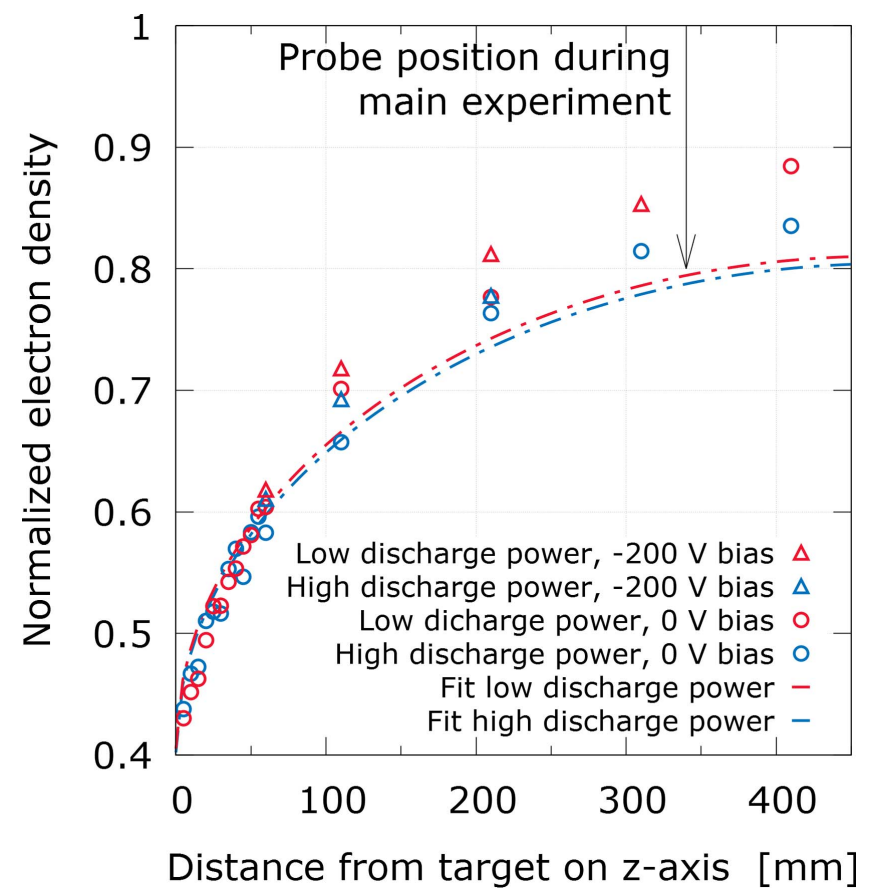

Figure 2. Decay of the electron density in the pre sheath estimated by moving the target plate stepwise towards the Langmuir probe. At the same time the electron temperature stays unchanged.

Table 1. Plasma parameters and decay length of the $400.9 \mathrm{~nm} \mathrm{~W} \mathrm{I}$ line for cases $\mathrm{A}-\mathrm{E}$ as shown in figure 3 .

\begin{tabular}{ccccc}
\hline & $\begin{array}{c}\text { Decay } \\
\text { length }(\mathrm{mm})\end{array}$ & $T_{e}(\mathrm{eV})$ & $n_{e}\left(\mathrm{~m}^{-3}\right)$ & $\begin{array}{c}\text { Discharge } \\
\text { power }(\mathrm{kW})\end{array}$ \\
\hline $\mathrm{A}$ & 48 & 6.2 & 0.2 & 3.6 \\
$\mathrm{~B}$ & 40 & 6.7 & 0.6 & 5.1 \\
$\mathrm{C}$ & 33 & 8.2 & 0.9 & 6.7 \\
$\mathrm{D}$ & 27 & 8.5 & 1.2 & 8.0 \\
$\mathrm{E}$ & 21 & 10.0 & 1.6 & 15.8 \\
\hline
\end{tabular}

placed $34 \mathrm{~cm}$ from the target plate. However, between the probe position and the target plate the electron density drops significantly due to the pre sheath. This drop is quantified for two representative plasma scenarios by moving the target plate stepwise towards the probe position (figure 2). With respect to the electron temperature no relevant dependency on the probe position is observed.

\section{Experimental data and analysis}

We observe the decay behaviour of various W I spectral lines in front of the target plate for a number of pure neon plasma scenarios that mainly differ in the peak values of the electron density and temperature trying to cover the widest possible range of ionisation rates $S=n_{e} \cdot\left\langle\sigma_{\text {ion }} \cdot v\right\rangle\left(T_{e}\right)$. For data analysis we interpret the intensity $I(z)$ of each of the spectral lines as exponential decay and obtain the experimental decay length $\lambda_{\exp }$ by fitting the expression $I(z) \propto \exp \left(-z / \lambda_{\exp }\right)$ to the data (figure 3$)$. The fit only takes into account $z$-values 


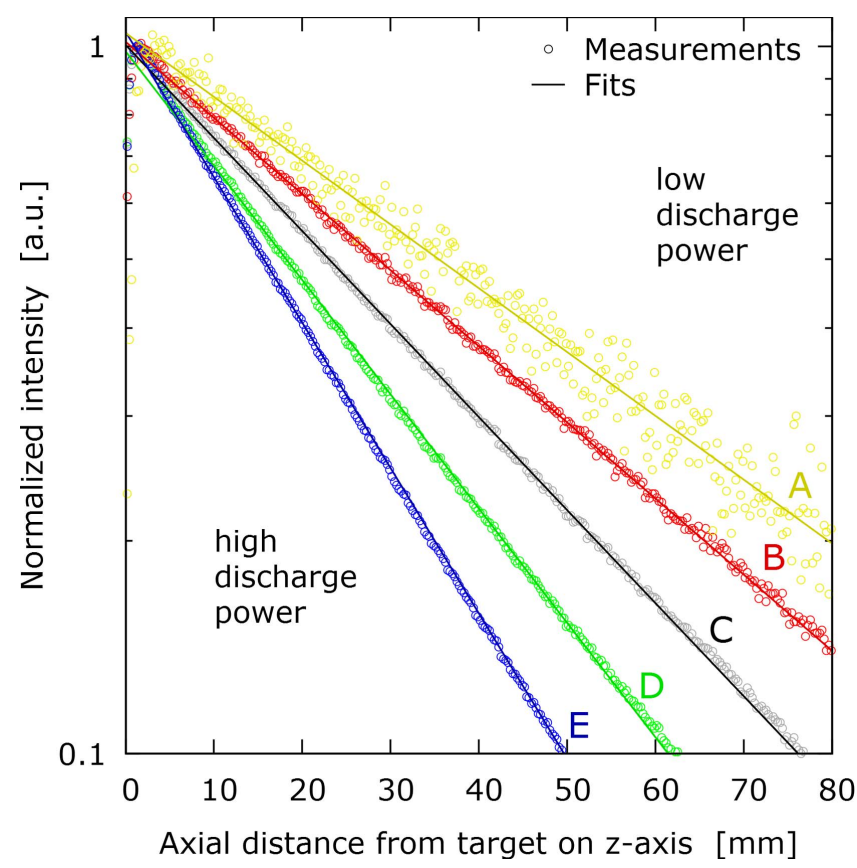

Figure 3. Decay length of the $400.9 \mathrm{~nm}$ line of W I observed in neon plasmas with different discharge powers. The plasma parameters of cases A-E are given in table 1.

between $20-60 \mathrm{~mm}$ safely excluding the intensity peak close to the target plate as well as the region very far from the target plate where the decay starts to deviate from the exponential behavior due to geometrical effects ${ }^{1}$. The resulting $\lambda_{\exp }$ for different W I spectral lines plotted against the inverse ionisation rate are shown on the left side of figure 4 . For each of the spectral lines we find a constant maximum decay length at very low ionisation rates that significantly decreases towards higher ionisation rates (i.e. towards higher discharge powers). This observation is described by the simple expression:

$$
\lambda_{\exp }=\left(\frac{1}{\lambda_{\text {ion }}}+\frac{1}{\lambda_{g}}\right)^{-1}=\left(n_{e} \cdot \frac{\left\langle\sigma_{\mathrm{ion}}\right\rangle \cdot v\left(T_{e}\right)}{\left\langle v_{w}\right\rangle}+\frac{1}{\lambda_{g}}\right)^{-1},
$$

where $\lambda_{g}$ is the (supposedly constant) maximum decay length caused by geometrical effects only and $\left\langle v_{w}\right\rangle$ is the mean velocity of the sputtered $\mathrm{W}$ atoms. We fit expression 2 to the experimental data using $\lambda_{g}$ and $\left\langle v_{w}\right\rangle$ as free parameters (figure 4 , left side). Of course, the resulting value of $\left\langle v_{w}\right\rangle$ depends on the employed data set for $\left\langle\sigma_{\text {ion }} \cdot v\right\rangle\left(T_{e}\right)$ while $\lambda_{g}$ is almost independent. Surprisingly though, the $\lambda_{g}$ obtained from the different W I spectral lines vary between 45 and $75 \mathrm{~mm}$. This cannot be connected to differences in the ionisation rates from different $\mathrm{W}$ I levels as ionisation should not play a role at low discharge powers where $\lambda_{\exp }=\lambda_{g}$. The effect might, however, be explainable by differences in the rate coefficients for electron impact excitation. With respect to $\left\langle v_{w}\right\rangle$ the fit results in values between $8.0 \times 10^{3}$ and $1.4 \times$ $10^{4} \mathrm{~m} \mathrm{~s}^{-1}$ when assuming the ionisation data by Vainstein et al. This is much higher than the velocities predicted by the

1 This behavior is qualitatively confirmed by ERO modelling (see [7]). In [17] a similar observation is reported from the linear plasma device PISCES with different plasma conditions and geometries. established, semi empirical Thompson formula for the energy distribution of sputtered particles $[10,11]$ :

$$
f(E) \mathrm{d} E=E \cdot \frac{\left(1-\frac{\left(E+E_{i z}\right)}{\gamma \cdot E_{p}}\right)}{\left(E+E_{i z}\right)^{1+n}} \quad \text { with } \gamma=4 \cdot \frac{m_{1} \cdot m_{2}}{m_{1}^{2}+m_{2}^{2}},
$$

where for our set up $E_{p}=200 \mathrm{eV}, \quad m_{1}=20 \mathrm{u}$ and $m_{2}=184 \mathrm{u}$. Following [11] we use $n=3.5$ leading to a reference mean velocity of approx. $2.8 \times 10^{3} \mathrm{~m} \mathrm{~s}^{-1}$ where the upper boundary of the integral was defined by the maximum transferrable energy during the sputter process. On the other hand, when employing the rate coefficient by Steinbrink fitting expression 2 to the data results in mean velocities of $2.0 \times 10^{3}-3.5 \times 10^{3} \mathrm{~m} \mathrm{~s}^{-1}$ that match the Thompson theory much better.

Given the knowledge of $\left\langle v_{w}\right\rangle$ one can determine the rate coefficient for ionisation of $\mathrm{W}$ I by rearranging expression 2 :

$$
\left\langle\sigma_{\text {ion }} \cdot v\right\rangle\left(T_{e}\right)=\frac{\left\langle v_{w}\right\rangle}{n_{e}} \cdot\left(\frac{1}{\lambda_{\exp }}-\frac{1}{\lambda_{g}}\right) .
$$

However, without a detailed analysis of $\left\langle v_{w}\right\rangle$ one canstrictly speaking-only experimentally access the quantity $\left\langle\sigma_{\text {ion }} \cdot v\right\rangle /\left\langle v_{w}\right\rangle$, which is shown on the right side of figure 4 for all data points with $\lambda_{\exp }<0.8 \cdot \lambda_{g}$. We still deduce results for the ionisation rate coefficient using the mean velocity as predicted by the Thomson model. The resulting $\left\langle\sigma_{\text {ion }} \cdot v\right\rangle$ averaged over all considered spectral lines are shown in figure 5 together with other data sets from literature. The present results compare well to the only other existing experimental data by Steinbrink [6], which were independently obtained using a different approach ${ }^{2}$. Both experimental data sets are, however, significantly lower than their widely used theoretical pendants based on the Born approximation (Vainstein [4]) or the the distorted wave approximation (ADAS [5], Pindzola and Griffin [13]). The same holds for the semi empirical formula by Lotz $[9,9 b]$. Only the more recent theoretical data by Goswamy et al [14] calculated using the spherical complex optical potential formalism combined with the complex scattering potential ionisation contribution method are closer by the experimental results, although they do not confirm the temperature dependence of the Steinbrink data.

Additionally, the experiment was modelled with the Monte Carlo Code ERO [15, 16]. ERO calculates the 3D density distribution for all relevant $\mathrm{W}$ ion stages and then simulates the emission profiles for our experimental set up (virtual diagnostic) using the photo emission coefficient for the $400.9 \mathrm{~nm}$ spectral line from [5]. For comparison the ERO data is then handled the same way as the experimental data. Figures 6(a) and (b) show that the ERO modelling achieves better agreement with the experimental findings when using the Steinbrink data compared to the Vainstein data. The remaining offset between the modelling and experimental

2 The $400.9 \mathrm{~nm} \mathrm{~W}$ I spectral line was measured in front of a $1 \times 1 \mathrm{~cm}^{2} \mathrm{~W}$ target plate in the linear plasma device PSI-1 with spatial resolution in $z$-direction. The observed line intensity $I(z)$ was modelled by means of the angular distribution of the sputtered $\mathrm{W}$ atoms and the electron impact ionisation of the $\mathrm{W}$ atoms. 

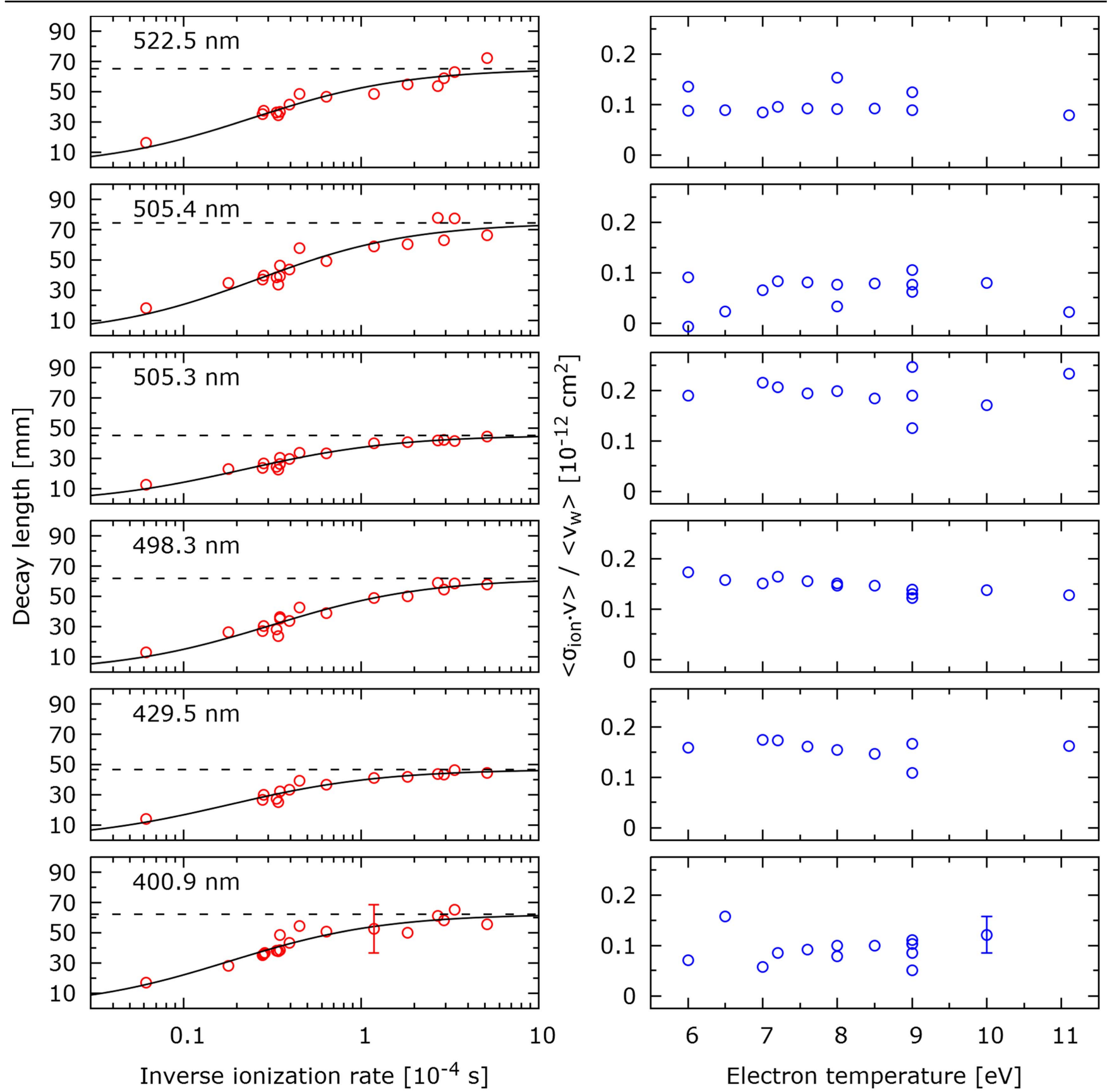

Figure 4. Decay lengths of different $\mathrm{W}_{\mathrm{I}}$ lines (left) and the deduced quantity $\left\langle\sigma_{\text {ion }} \cdot v\right\rangle /\left\langle v_{w}\right\rangle$ (right).

results may e.g. be due to deviations from the angular distribution of the sputtered particles assumed in the ERO calculations.

So far only the decay behaviour of different W I spectral lines was discussed. However, a look at the swing in behaviour of these lines occurring very close to the target plate reveals differences to the behaviour earlier found at TEXTOR [17]. In TEXTOR the $400.9 \mathrm{~nm}$ line excited from the ${ }^{7} \mathrm{~S}_{3}$ state reached its intensity peak significantly later than those lines excited from the ${ }^{5} \mathrm{D}_{J}$ multiplet. This is not the case in PSI-2 plasmas. Here the $400.9 \mathrm{~nm}$ line is among the first to peak. We consider this strong evidence against a recently discussed hypothesis, according to which the metastable ${ }^{7} \mathrm{~S}_{3}$ state is generally populated much slower than the ${ }^{5} \mathrm{D}_{J}$ states. This observation rather speaks for differences in the rate coefficients for the excitation of these lines that do play a role at temperatures of approx. $50-100 \mathrm{eV}$ as present in the TEXTOR edge plasma, but not at the much lower temperatures of approx. 5-10 eV reached in PSI-2.

\section{Conclusion}

Currently, extensive experiments are performed at different plasma devices in order to predict the lifetime of plasmafacing components made of tungsten in future fusion devices. In these experiments the sputtered neutral tungsten atoms are observed spectroscopically. At the same time the WI emission 


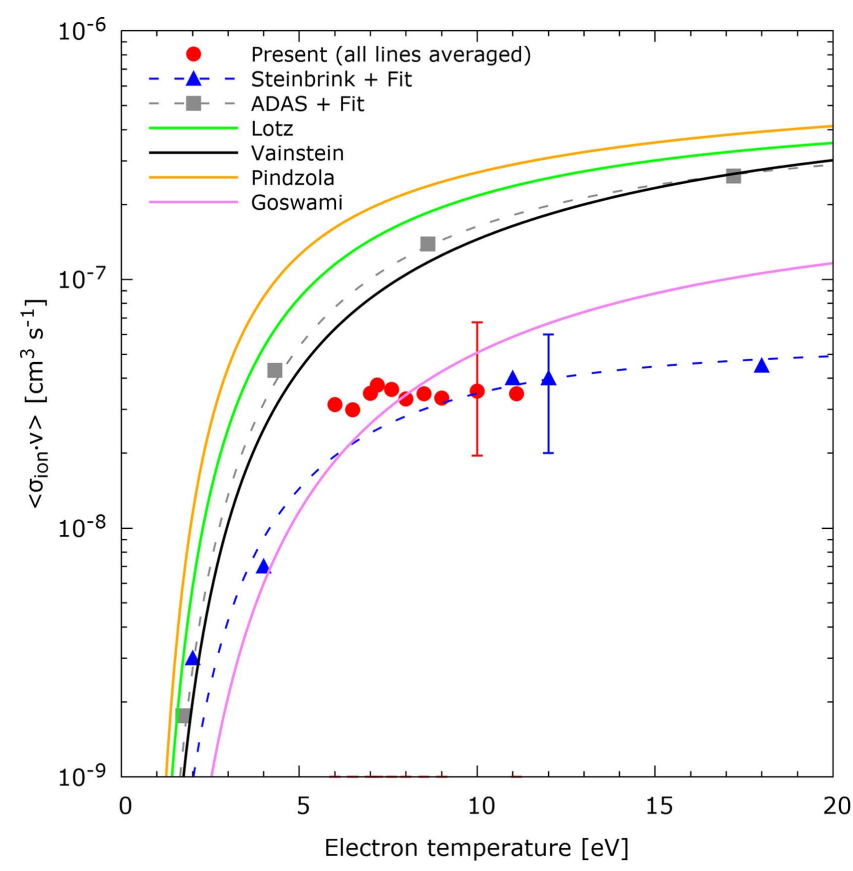

Figure 5. Available experimental and theoretical data sets on the rate coefficient for electron impact ionisation of $\mathrm{W}$.

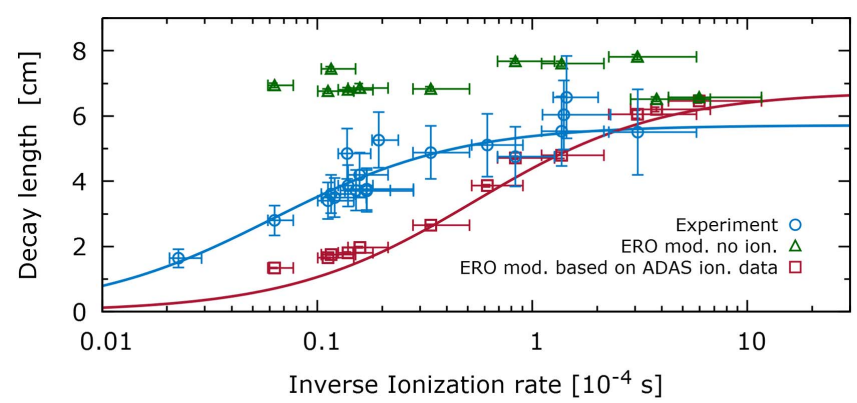

(a)

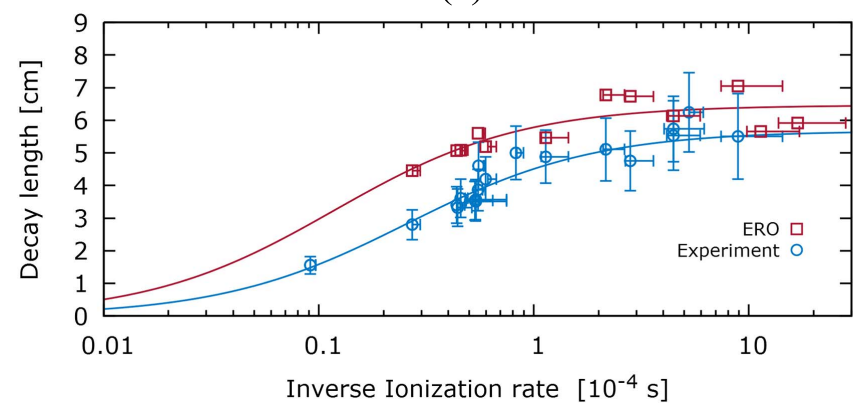

(b)

Figure 6. Decay length of the $400.9 \mathrm{~nm}$ line of W I as a function of the inverse ionisation rate. Blue circles are experimental data, red squares are ERO modelling results and green triangles are ERO modelling results with artificially switched off ionisation. Based on ionisation data by Vainstein (a) and by Steinbrink (b).

is modelled with plasma-surface interaction codes like ERO. In this respect the atomic data on electron impact ionisation and excitation are critical-both for the interpretation of the WI emission profiles as well as for the modelling. We present experimental results on the rate coefficient for electron impact ionisation of $\mathrm{W}$ atoms for near threshold temperatures. The present data confirm earlier experimental results [6] that were independently obtained using a different method. The experimental data sets are, however, significantly lower than widely used theoretical data sets based on the Born [4] and the distorted wave approximation [5, 13]. Better agreement in absolute values is found for more recent calculations by Goswami et al [14]. Within our evaluation the two experimental data sets are consistent with the energy distribution of the sputtered $\mathrm{W}$ atoms predicted by the established semi empirical law by Thompson [10, 11], while the theoretical data sets are not.

The method applied can easily be extended to other target materials to access other elements where data are scarce or not available at all, while a wider range of electron temperatures can be covered by using different working gases. Possible improvements of the method include the spectroscopic analysis of the energy and angular distribution of the sputtered particles as well as the simultaneous observation of W II spectral lines where visible. It might further be of interest to study a possible impact of the target geometry. For the present work we used a big target plate covering the entire plasma diameter in order to improve the signal to noise ratio. In principle though the target should be small against the plasma dimension to avoid possible sources of error. First tests with a small target showed significantly reduced decay lengths of the W I spectral lines, which we assign to geometrical effects and is confirmed by ERO modelling. The decay length's dependency on the discharge power, however, seemed to stay unchanged so that we expect similar results. This is further supported by the good agreement of our data with Steinbrink's results that were obtained with a small $1 \times 1 \mathrm{~cm}^{2} \mathrm{~W}$ target.

Finally, it shall be mentioned that the applied method should allow for a quantification of the volumetric losses of sputtered particles relative to the losses caused by ionisation via the ratio $\lambda_{\text {ion }} / \lambda_{g}$. This is important for $\mathrm{S} / \mathrm{XB}$-based flux measurements in weakly ionising plasmas, where the volumetric losses tend to be in the same order of magnitude or even much higher than the losses due to ionisation so that a corrective term becomes indispensable.

\section{Acknowledgments}

This work has been carried out within the framework of the EUROfusion Consortium and has received funding from the Euratom research and training programme 2014-2018 under grant agreement No. 633053. The views and opinions expressed herein do not necessarily reflect those of the European Commission. This work was done under WP PFC.

\section{ORCID iDs}

S Brezinsek (iD https://orcid.org/0000-0002-7213-3326

A Kirschner (10) https://orcid.org/0000-0002-3213-3225

Ch Linsmeier (10) https://orcid.org/0000-0003-0404-7191 


\section{References}

[1] Brezinsek S et al $2015 \mathrm{~J}$. Nucl. Mater. 46311

[2] Pütterich T et al 2013 Plasma Phys. Control. Fusion 55 124036

[3] Brezinsek S et al 2011 Phys. Scr. T145 014016

[4] Vainshtein L et al 2011 J. Phys. B: At. Mol. Opt. Phys. 44 125201

[5] ADAS http://open.adas.ac.uk

[6] Steinbrink J 1997 Diploma Thesis Humboldt-Universität Berlin

[7] Ertmer S 2017 Master Thesis Universität Düsseldorf

[8] Kreter A et al 2015 Fus. Sci. Technol. 6888
[9] Behrisch R and Eckstein W 2007 Sputtering by particle bombardment: experiments and computer calculations from theshold to MeV energies Top. Appl. Phys. 110

Naujoks D et al 1996 Nucl. Fusion 36 671-87

[10] Thompson M W 1968 Phil. Mag. 18377

[11] Bay H L and Schweer B 1985 Jül-2032

[12] Lotz W 1970 Z. Phys. 232101

[13] Pindzola M S and Griffin D C 1992 Phys. Rev. A 462486

[14] Goswami B 2014 Int. J. Mass Spectrom. 3728

[15] Marenkov E et al 2015 J. Nucl. Mater. 463268

[16] Eksaeva A A et al 2016 J. Nucl. Mater. in preparation

[17] Längner M et al 2013 J. Nucl. Mater. 438865

[18] Nishijima D et al 2009 Phys. Plasmas 16122503 